\title{
Numerical modelling of thermoelastic processes using nonlinear theories with thermal relaxation time
}

D. V. Strunin*

R. V. N. Melnik ${ }^{\dagger}$

A. J. Roberts*

(Received 7 August 2000)

\begin{abstract}
A nonlinear model of thermoelasticity is solved for a ring-shaped body with special attention given to the effect of relaxation time on

* Department of Mathematics and Computing, University of Southern Queensland, Toowoomba, Queensland 4350, AustraliA. mailto:strunin@usq.edu. au and mailto: aroberts@usq. edu . au

$\dagger$ CSIRO Mathematical and Information Sciences, Macquarie University Campus, North Ryde, NSW 2113, AustraliA. mailto:Roderick.Melnik@cmis.csiro.au

${ }^{0}$ See http: //anziamj . austms . org. au/V42/CTAC99/Stru for this article and ancillary services, (C) Austral. Mathematical Soc. 2000. Published 27 Nov 2000.
\end{abstract}


the body dynamics. A spectral Galerkin method is used to accurately resolve spatial structures of temperature and displacement. A sequence of solutions is obtained for gradually increasing initial amplitude of the thermoelastic wave, showing a transition from a steady harmonic wave to singular solutions. Larger values of the relaxation time are shown to result in smoother temperature profiles. The dynamics generated by a localised heat release from an external source is studied. It is observed that the source excites a standing-like wave of the displacement while temperature monotonically decays.

\section{Contents}

1 Introduction: coupled dynamic thermoelasticity with relaxation times

C1358

2 Governing equations and the discretisation procedure

C1362

3 Numerical experiments: effects of nonlinearities and relaxation times

C1366

4 Conclusions

C1374

References

C1375 
1 Introduction: coupled dynamic thermoelasticity with relaxation times C1358

\section{Introduction: coupled dynamic thermoe- lasticity with relaxation times}

The classical Fourier law of heat conduction and consequent mathematical models for temperature dynamics constructed on the basis of parabolic partial differential equations assume instantaneous propagation of thermal disturbances to infinitely remote regions. Such an assumption is physically unrealistic and, in a number of practically important situations, is inadequate as a description of heat conduction $[2,3,16]$. A finite speed of thermal disturbances can be taken into account by using models with thermal relaxation time, which are based on hyperbolic-type equations for temperature. Those are closely connected with so-called theories with second sound which view heat propagation as a wave-like phenomenon.

The literature dedicated to the hyperbolic thermoelastic models is quite large and its detailed review can be found in $[2,3]$. However, the majority of the works in this field has been devoted to various aspects of linear models with some noticeable exceptions such as [10,8], where simplified (elastically linear) nonlinear models have been considered, and [18, 19, 12], where wellposedness issues have been studied.

Our aim in this paper is the numerical study of the fully nonlinear system of coupled thermoelasticity and the investigation of the influence of the relaxation time on the nonlinear dynamic of thermomechanical systems. Most of the previous contributions to the development of nonlinear models of ther- 


\section{Introduction: coupled dynamic thermoelasticity with relaxation times C1359}

moelasticity (including the above cited papers [18, 19, 12]) have been concentrated on the important theoretical issues such as existence and uniqueness of solutions, and the identification of conditions leading to smooth or singular solutions. Only few papers report numerical results obtained with nonlinear models of coupled thermoelasticity (see [1, 9, 20] and references therein). Using simple sinusoidal waves as initial conditions, in this paper we demonstrate some important features of such models by the example of ring-shaped thermoelastic bodies. Our main results concern the nonlinear interaction between thermal and mechanical fields and the effect of relaxation time.

It is well known $[13,15$, e.g.] that the simple nonlinear equation for a longitudinal displacement $u$ in an elastic bar $\partial^{2} u / \partial t^{2}=\partial / \partial x[\sigma(\partial u / \partial x)]$ ( $\sigma$ is the $\partial u / \partial x$-dependent stress) may lead to a singular solution even for smooth initial data. However, in the case of coupled thermoelasticity the formation of singularities may be suppressed by the dissipative mechanism of heat conduction [21]. On the other hand, there may be the opposite situation where the damping through heat conduction is not strong enough to guarantee the smooth solution. Which of these situations occurs depends on the initial amplitudes of temperature and displacement and on the types of nonlinearities in governing equations.

In the literature addressing linear theories with relaxation times, most attention has been given to models formulated by Lord and Schulman (LS) [14] and Green and Lindsay (GL) [7]. Both models are encompassed by a single system of equations which is written here for the case of homogeneous 


\section{Introduction: coupled dynamic thermoelasticity with relaxation times $\mathrm{C} 1360$}

isotropic medium:

$$
\left\{\begin{array}{l}
\rho \frac{\partial^{2} \mathbf{u}}{\partial t^{2}}-\mu \Delta \mathbf{u}-(\lambda+\mu) \nabla \nabla \mathbf{u}-\beta\left(1+t_{1} \frac{\partial}{\partial t}\right) \nabla \theta=0, \\
\rho c\left(t_{2}+t_{0}\right) \frac{\partial^{2} \theta}{\partial t^{2}}+\rho c \frac{\partial \theta}{\partial t}-K \triangle \theta-\beta T_{0}\left(\nabla \frac{\partial \mathbf{u}}{\partial t}+t_{0} \nabla \frac{\partial^{2} \mathbf{u}}{\partial t^{2}}\right)=0
\end{array}\right.
$$

where $\theta$ is the departure of the temperature from some reference value $T_{0}, \rho$ is the density of the material, $c$ is the thermal capacity, $t_{0}, t_{1}$ and $t_{2}$ are the thermal relaxation times, $\mu$ and $\lambda$ are Lamé coefficients, $K$ is the coefficient of heat conduction, $\beta$ is the coefficient of thermal pressure.

The LS model follows from (1) by setting $t_{1}=t_{2}=0$. Alternatively, if we set $t_{0}=0$ in (1) we obtain the GL model. The linear hyperbolic models such as (1) have been intensively studied $[22,21,11,5,17,6]$.

The model (1) can be generalised to include nonlinear terms: $\mathbf{F}_{1}(\tilde{\nabla} \mathbf{u}$, $\nabla \tilde{\nabla} \mathbf{u}, \tilde{\nabla} \theta, \nabla \tilde{\nabla} \theta)$ and $F_{2}(\tilde{\nabla} \mathbf{u}, \nabla \tilde{\nabla} \mathbf{u}, \tilde{\nabla} \theta, \nabla \tilde{\nabla} \theta)$ in the right-hand sides of the first and second equations respectively. The operator $\tilde{\nabla}$ is defined as $\tilde{\nabla} \equiv$ $(\partial / \partial t, \nabla)$. Nonlinearities represented by the functions $\mathbf{F}_{1}$ and $F_{2}$ are determined by the constitutive relations that couple stresses, deformation gradients (strains), temperature and heat fluxes.

Using simple polynomial forms of the functions $\mathbf{F}_{\mathbf{1}}$ and $F_{2}$ and a special choice of boundary conditions, the authors of the recent works [1, 20] studied one-dimensional nonlinear problems of thermoelasticity. No relaxation time effects were taken into account. In our paper we consider a more general 


\section{Introduction: coupled dynamic thermoelasticity with relaxation times C1361}
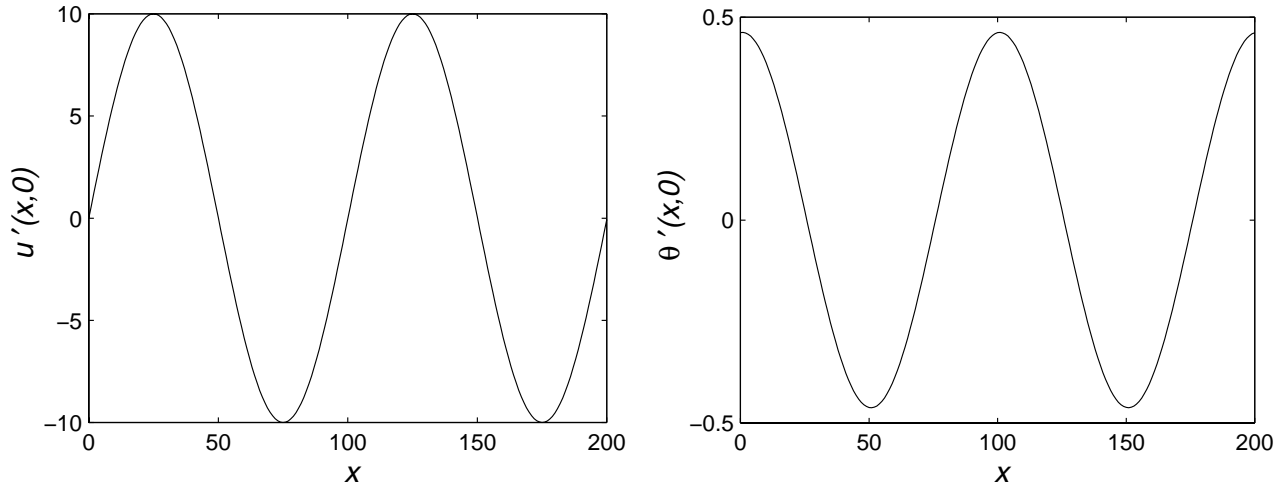

Figure 1: Steady wave solutions used as initial conditions for the nonlinear problem (2) in the first and the third groups of experiments. 
situation allowing for the relaxation-time effects.

Various thermomechanical boundary dissipation mechanisms have been proposed in the literature to obtain the (uniform) stability of the overall system [12, e.g.]. By considering a "closed" system in ring-shaped form, in this paper we follow a somewhat different direction. On the one hand, such a consideration allows us to avoid in our analysis unnecessary complicated (and often physically unrealistic) boundary conditions. From a formal point of view, one can view our system as a thermomechanical system subjected to periodic boundary conditions, satisfied automatically by the Fourier series form of the solution. On the other hand, ring-shaped configurations are quite common elements in engineering devices. Finally, the ring is a very convenient object for theoretical study of the thermoelastic dynamics free from external influences. In this case the boundary thermomechanical dissipation is absent and we do not have to deal with issues discussed, for example, in $[12]$.

\section{Governing equations and the discretisation procedure}

We consider the model obtained by a "superposition" of the linear GL model and the nonlinear model [1]. This model is a natural generalisation of the socalled monomode nonlinear model to the case of non-zero relaxation times. 
A non-dimensional form of this model is obtained by using the scales (see notation for the systems (1) and (2))

$$
\bar{u}=\frac{K}{c \sqrt{\rho(\lambda+2 \mu)}}, \quad \bar{\theta}_{*}=T_{0}, \quad \bar{x}=\frac{K}{c \sqrt{\rho(\lambda+2 \mu)}}, \quad \bar{t}=\frac{K}{c(\lambda+2 \mu)},
$$

and defining non-dimensional parameters

$$
\tau_{1}=\frac{t_{1} c(\lambda+2 \mu)}{K}, \quad \tau_{2}=\frac{t_{2} c(\lambda+2 \mu)}{K}, \quad \beta_{1}=\frac{\beta T_{0}}{\lambda+2 \mu}, \quad a=\frac{\beta}{\rho c} .
$$

We have

$$
\left\{\begin{aligned}
& \frac{\partial^{2} u}{\partial t^{2}}-\frac{\partial^{2} u}{\partial x^{2}}\left(1+2 \gamma \frac{\partial u}{\partial x}+3 \delta\left(\frac{\partial u}{\partial x}\right)^{2}\right) \\
&- \beta_{1} \frac{\partial \theta}{\partial x}-\beta_{1} \tau_{1} \frac{\partial^{2} \theta}{\partial x \partial t}-\beta_{2} \frac{\partial}{\partial x}\left(\frac{\partial u}{\partial x} \theta\right)=0 \\
& \tau_{2} \frac{\partial^{2} \theta}{\partial t^{2}}+\frac{\partial}{\partial t}\left(\theta-a \frac{\partial u}{\partial x}-\frac{1}{2} b\left(\frac{\partial u}{\partial x}\right)^{2}\right)-\frac{\partial}{\partial x}\left(\left(1+\alpha \frac{\partial u}{\partial x}\right) \frac{\partial \theta}{\partial x}\right)=0 .
\end{aligned}\right.
$$

For simplicity, hereafter we use the same notations for the dimensionless quantities as we used earlier for the dimensional ones. The nondimensional values $\gamma, \delta, \beta_{1}, \beta_{2}, a, b$ and $\alpha$ are assumed constants. We consider the thermomechanical body in the form of a ring characterised by a base wave number $k=2 \pi / L$, where $L$ is the perimeter of the ring. 
Introduce the field of velocity and the rate of temperature change:

$$
v=\frac{\partial u}{\partial t}, \quad w=\frac{\partial \theta}{\partial t} .
$$

Then we seek the solution to (2) in the form of Fourier series

$$
\begin{array}{ll}
u=\sum_{n=-\infty}^{\infty} U_{n}(t) e^{i n k x}, & v=\sum_{n=-\infty}^{\infty} V_{n}(t) e^{i n k x}, \\
\theta & =\sum_{n=-\infty}^{\infty} \Theta_{n}(t) e^{i n k x}, \quad w=\sum_{n=-\infty}^{\infty} W_{n}(t) e^{i n k x} .
\end{array}
$$

Substituting (3) into (2) and equating coefficients of $\exp ($ inkx) leads to an infinite system of coupled ordinary differential equations for the amplitudes of the Fourier modes. This system is truncated to a finite number of modes, typically 32, and integrated in time using the fourth order Runge-Kutta method. The numerical scheme may be regarded as the spectral Galerkin method with harmonical basis functions. A number of test runs were organised in the following way.

The equations (2) were solved with such forcing functions $f(x, t)$ and $g(x, t)$ that provide the prescribed analytical form of the solution. Then the analytical and numerical solutions were compared with each other. For example, a test was performed with the exact solution $u=(1+A / 3 \sin (k x-\omega t))^{N}$, $\theta=C \cos (k x-\omega t)$ for constant integer $N$ and constants $A$ and $\omega$. Having substituted these expressions into (2), we calculated the functions $f(x, t)$ and 
$g(x, t)$. Note that the cubic nonlinearity in (2) generates $3 N$ modes after decomposition into the Fourier series. Consequently, the functions $f(x, t)$ and $g(x, t)$ are quite cumbersome even for the case $N=3$ being composed of $3 N=9$ modes. As we have both cosines and sines in the series, this gives 18 terms.

In addition to the forced nonlinear regimes characterised by different forms of the desired solution, the numerical code reproduced quite well an exact travelling wave solution of the unforced linearised system (2) with $\beta_{1}=\gamma=\delta=\beta_{2}=b=\alpha=0, a \neq 0$ (note that a similar wave was also used in [1] where, however, no relaxation time was taken into account):

$$
\begin{gathered}
u=A \sin (k(x-t)), \\
\theta=\frac{\left(1-\tau_{2}\right) a A k^{2}}{\left(1-\tau_{2}\right)^{2} k^{2}+1} \sin (k(x-t))+\frac{a A k}{\left(1-\tau_{2}\right)^{2} k^{2}+1} \cos (k(x-t)) .
\end{gathered}
$$

Figure 1, where both the numerical solution and exact solution (4) are presented for $A=10.0, a=0.926, k=0.05, \tau_{2}=0.005$, confirms that there is no discrepancy between the numerical and exact solutions. In this graph and the other graphs below we indicate the coordinate $x$ in conditional units to show the number of points used to plot the curves. In the next section we use (4) to set the initial conditions for the first series of experiments with the nonlinear problem (2). Note that the wave (4) represents asymptotically neutral state of the system in which the mechanical field is decoupled from the thermal field. 
3 Numerical experiments: effects of nonlinearities and relaxation times C1366
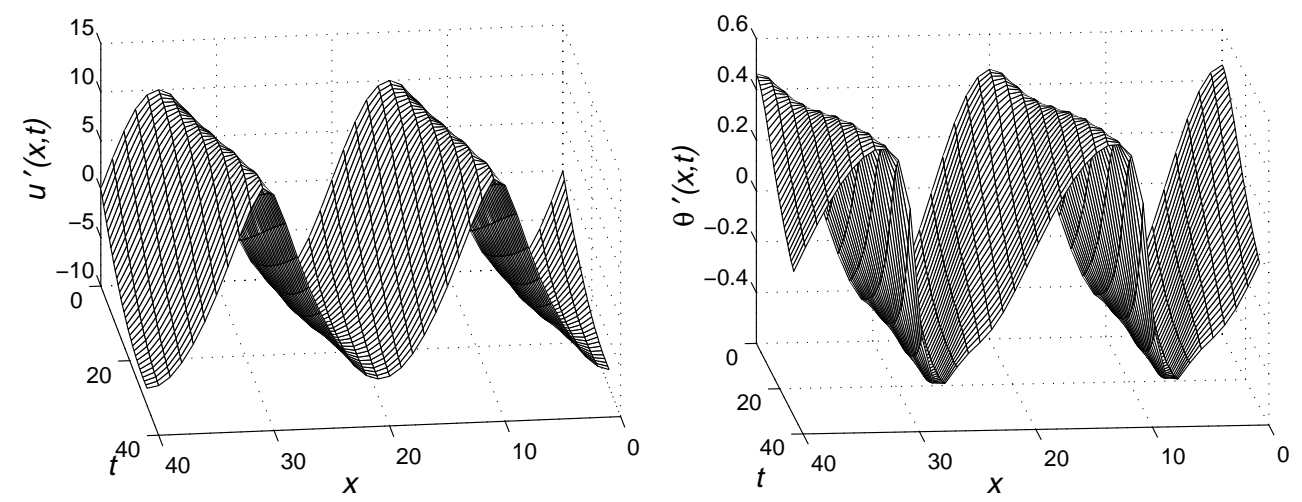

Figure 2: Displacement and temperature dynamics in the weakly nonlinear case $\varepsilon=1.0$.

\section{Numerical experiments: effects of nonlin- earities and relaxation times}

The first group of experiments examines the dynamics of the thermomechanical system excited by the initial wave (4) as the amplitude $A$ is increased and, consequently, the nonlinear effects become more important. One of the difficulties here stems from the hyperbolic nature of the problem. Indeed, in this case the set of initial conditions includes initial values of time-derivatives of temperature and of displacements. To overcome this difficulty we assume that these initial values can be altered proportionally to the initial amplitudes 


\section{Numerical experiments: effects of nonlinearities and relaxation times C1367}
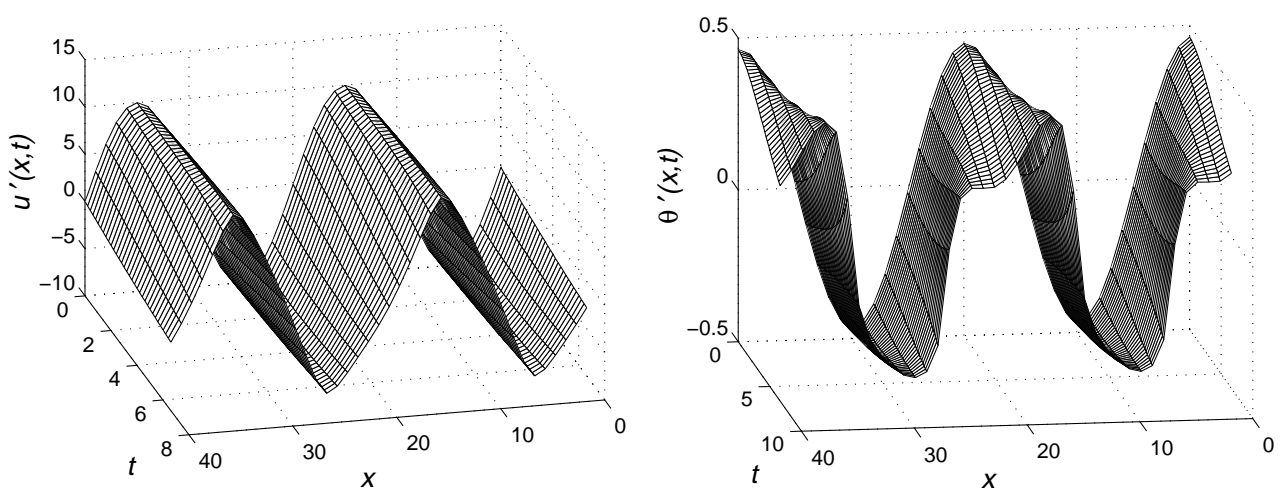

Figure 3: Displacement and temperature dynamics in the moderately nonlinearcase $\varepsilon=5.0$.

which are changed from experiment to experiment.

Using this assumption, our numerical experiments were organised in such a way that, instead of increasing the initial amplitudes, we gradually increased the coefficients near the nonlinear terms. To determine how the coefficients must change, we replace the variables $u, \theta$ by new variables marked by primes, $u^{\prime}, \theta^{\prime}$, and defined by $u=\varepsilon u^{\prime}, \theta=\varepsilon \theta^{\prime}$, where $\varepsilon$ is a positive scaling 


\section{Numerical experiments: effects of nonlinearities and relaxation times C1368}

factor. This leads to a system for $u^{\prime}$ and $\theta^{\prime}$ :

$$
\left\{\begin{array}{c}
\frac{\partial^{2} u^{\prime}}{\partial t^{2}}-\frac{\partial^{2} u^{\prime}}{\partial x^{2}}\left(1+2 \gamma \varepsilon \frac{\partial u^{\prime}}{\partial x}+3 \delta \varepsilon^{2}\left(\frac{\partial u^{\prime}}{\partial x}\right)^{2}\right) \\
-\beta_{1} \frac{\partial \theta^{\prime}}{\partial x}-\beta_{1} \tau_{1} \frac{\partial^{2} \theta^{\prime}}{\partial x \partial t}-\beta_{2} \varepsilon \frac{\partial}{\partial x}\left(\frac{\partial u^{\prime}}{\partial x} \theta^{\prime}\right)=0 \\
\tau_{2} \frac{\partial^{2} \theta^{\prime}}{\partial t^{2}}+\frac{\partial}{\partial t}\left(\theta^{\prime}-a \frac{\partial u^{\prime}}{\partial x}-\frac{1}{2} b \varepsilon\left(\frac{\partial u^{\prime}}{\partial x}\right)^{2}\right) \\
-\frac{\partial}{\partial x}\left(\left(1+\alpha \varepsilon \frac{\partial u^{\prime}}{\partial x}\right) \frac{\partial \theta^{\prime}}{\partial x}\right)=0
\end{array}\right.
$$

The systems (2) and (5) differ only in the values of the coefficients. As is evident from (5), the scaled coefficients which we mark by primes are

$$
\gamma^{\prime}=\varepsilon \gamma, \quad \delta^{\prime}=\varepsilon^{2} \delta, \quad \beta_{1}^{\prime}=\beta_{1}, \quad \beta_{2}^{\prime}=\varepsilon \beta_{2}, \quad a^{\prime}=a, \quad b^{\prime}=\varepsilon b, \quad \alpha^{\prime}=\varepsilon \alpha .
$$

If $\varepsilon$ is infinitely small then the nonlinear terms are negligible compared to the linear terms. In the zero-order approximation in $\varepsilon$, we therefore recover the linear system. As we mentioned in Section 2, the linearised system (2) admits the solution (4). This solution represents a "steady" wave, or, more precisely, the wave that keeps its shape. This fact makes this wave an attractive candidatefor the initial conditions in our nonlinear problem. Choosing the initial conditions in the form of (4), we demonstrate below how the sinusoidal wave is deformed by the effects of nonlinearities. Any alterations in $\varepsilon$ not only lead to a change in the initial amplitudes of $(u, \theta)$, but also to the proportional 

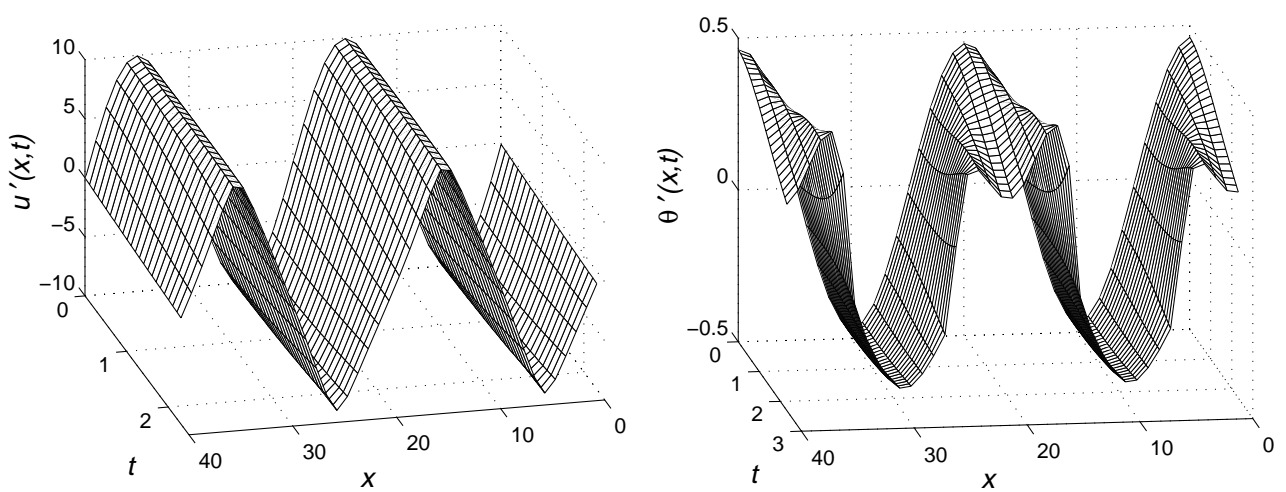

Figure 4: Displacement and temperature dynamics in the strongly nonlinear case $\varepsilon=15.0$.

change in their time-derivatives. Thus, depending on the magnitude of $\varepsilon$ we can obtain a range of initial conditions for our problem in terms of $(u, \theta)$. Furthermore, by increasing $\varepsilon$, we can amplify the influence of nonlinear effects in the thermoelastic system described by (5).

For sufficiently large values of $\varepsilon$, the nonlinearities come into action by dominating over the linear terms and leading to the formation of nonsmooth profiles. To demonstrate these nonlinear effects we present the solution of (5) in the form of 3-D graphs showing time evolution of the whole spatial profiles of displacement and temperature. Two periods of the profiles are plotted (thus, left-, middle- and right-hand points of each graph correspond to the same physical point). In our computations all coefficients near the linear 


\section{Numerical experiments: effects of nonlinearities and relaxation times C1370}
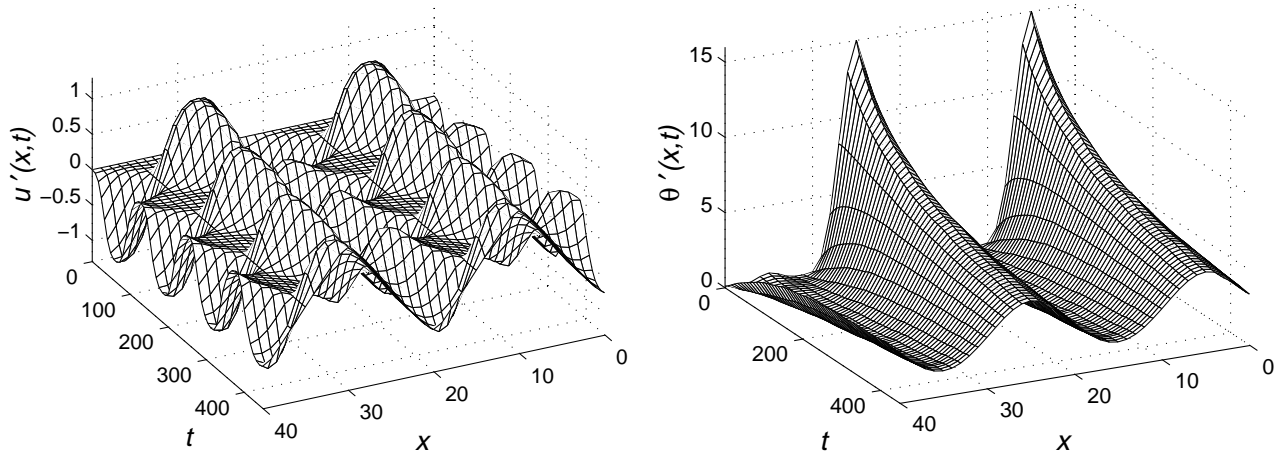

FIGURE 5: Dynamics produced by a pulse-like initial temperature.

terms in (5) were taken for steel, that is $a=0.926, \beta_{1}=5.035 \cdot 10^{-3}$. The rest of the coefficients were scaled respectively from the data presented in [1]: $\gamma=1.0, \delta=0.5, \beta_{2}=0.001, b=0.1, \alpha=1.0$. The reported results were obtained for 32 Fourier modes with the wave number $k=0.05$.

In Figure 2-4 we present the dynamics of the thermoelastic ring under different degrees of nonlinearity. The relaxation times for all three cases presented in Figure 2-4 were fixed at $\tau_{1}=\tau_{2}=\tau=0.005$. Figure $2(\varepsilon=1)$ demonstrates a sharpening of certain sections of the displacement and temperature profiles caused by nonlinearities. Both profiles gradually approach nonsmooth phases with discontinuous derivatives. Starting from sinusoidal waves shown in Figure 1, displacement and temperature distributions are transformed into distinctly different shapes according to the laws governing 


\section{Numerical experiments: effects of nonlinearities and relaxation times C1371}
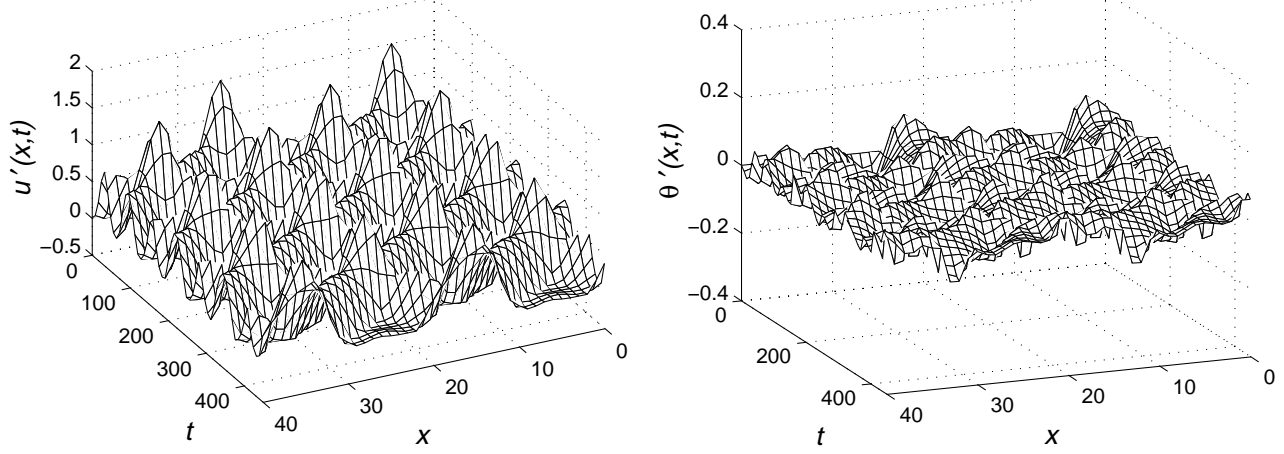

FiguRE 6: Dynamics produced by a pulse-like initial displacement.

their coupled dynamics. Figure $3(\varepsilon=5.0)$ and Figure $4(\varepsilon=15.0)$ demonstrate the increasing influence of the effects of nonlinearities. The nonlinear dynamics observed in this group of experiments has the following features. As demonstrated by Figure 3 and Figure 4, the main peak of the temperature distribution is split into two unequal peaks. In the meantime, with the increasing role of nonlinear effects the displacement profile assumes almost a triangular shape, as demonstrated in the strongly nonlinear case by Figure 4. Remarkably, with the nonlinear effects coming into play, the solution approaches the nonsmooth phase faster. Consequently, in order to avoid the rippled profiles indicating that the number of modes (32 in this work) is no longer sufficient to represent the solution accurately, we have to shorten the limiting time $t_{*}$ in our computational experiments. Since the existence of 
3 Numerical experiments: effects of nonlinearities and relaxation times $\mathrm{C} 1372$

smooth solutions for our mathematical model is limited by some finite interval of time $[1,13,19,20]$, these numerical difficulties are intrinsic to the nonlinear thermoelastic regimes under investigation.

The second group of experiments was organised as follows. First, using a pulse-like thermal and identical-zero mechanical initial conditions (Figure 5) and then, using a pulse-like mechanical and identical-zero thermal initial conditions (Figure 6), we studied the nonlinear interaction between thermal and mechanical fields. The scaling parameter was taken $\epsilon=1$, while all other parameters of the model remained unchanged. Figure 5 shows that the initial heat pulse brings about a standing-like elastic wave while temperature gradually fades. A more detailed analysis reveals that the displacement profile gradually sharpens under the action of nonlinearity. By contrast, the initial displacement-pulse generates substantially more complicated structures displayed in Figure 6. Elastic oscillations excite small-amplitude thermal waves and under the influence of nonlinearities their profile become sharper. Due to the coupling phenomenon, this complicates the thermal dynamics.

Finally, our last group of experiments deals with the influence of the relaxation time on the nonlinear dynamics of thermoelastic bodies. The range of reported values of relaxation times for different materials is quite wide, $10^{-15} \mathrm{~s}<t_{0}<10^{-10} \mathrm{~s}[2]$. When going from material to material, not only do $\tau_{1}$ and $\tau_{2}$ change but also so do all the other coefficients of the thermoelastic equations. Regrettably, among those, only the values of the coefficients of the linear terms in our model (5) are well tabulated in the literature, and no reliable data is available on the coefficients of the nonlinear terms. With 
3 Numerical experiments: effects of nonlinearities and relaxation times C1373
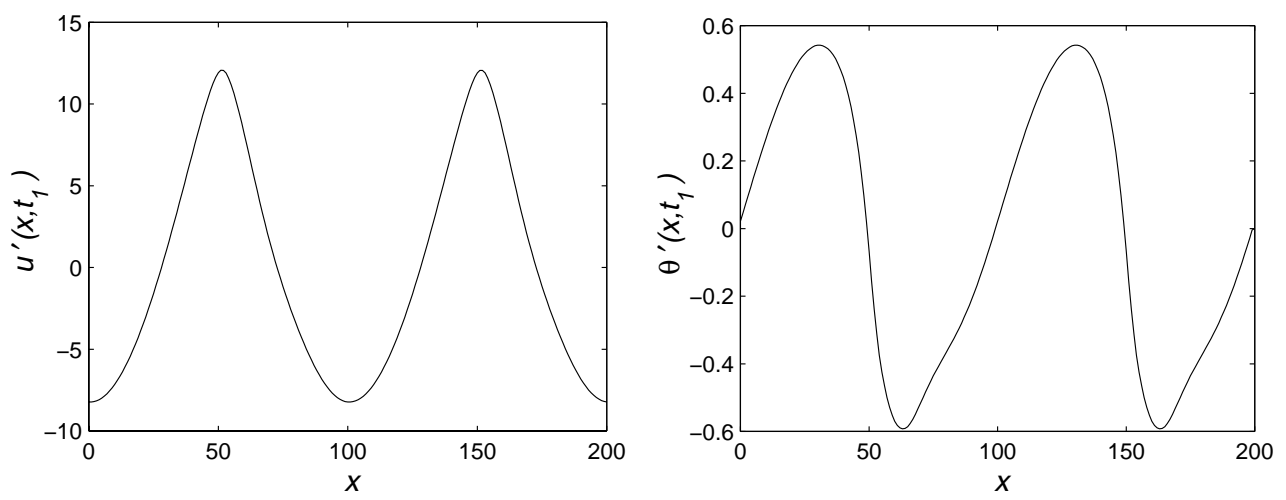

Figure 7: Displacement and temperature profiles at moment $t_{*}=32.5$ (the case of moderate relaxation times, $\tau=5.0)$.

this in mind, we explored how the relaxation time affects the solution provided that the rest of the coefficients are fixed. The initial conditions for this series of computations were again taken in the form shown in Figure 1. After the non-dimensionalisation based on the characteristics for steel, the above interval for $t_{0}$ converts into $5 \cdot 10^{-3}<\tau<5 \cdot 10^{2}$. In the series of numerical experiments we adopted $\tau_{1}=\tau_{2}=\tau$ and used fixed $\varepsilon=1$. Figure 7 shows the limiting profiles in the case of the relaxation time $\tau=5.0$. There are minor differences between these profiles and the profiles in Figure $2(\tau=0.005)$ corresponding to the same moment $t_{*}$. In both cases the temperature profiles are quite distorted by nonlinear effects accelarating the transition to the nonsmooth phase. A different situation is observed for 
large relaxation times. For example, in Figure 8 we present the result of computation for $\tau=500.0$, where the temperature profiles are nearly sinusoidal during the whole experiment. This confirms that larger relaxation times provide smoother temperature profiles formed by a fixed moment of time. To interpret this effect, recall that the major consequence of the relaxation-time effect is either fast (in the case where $\tau$ is small) or slow (in the case where $\tau$ is large) transitions of the thermomechanical system to certain regimes, typically characterised by solution discontinuities or nonsmooth phases of the solution with discontinuous derivatives. For larger $\tau$, transitions from the sinusoidal wave to the nonsmooth phase slow down. Figure 8 demonstrates that a large value of $\tau$ makes the transition extremely slow. In this case no sign of the formation of nonsmooth profiles is observed during the numerical experiment.

\section{Conclusions}

Using models with relaxation times, we analysed numerically nonlinear thermoelastic waves propagating through a ring. Starting with harmonic and pulse-like distributions for displacement and temperature, we conducted a series of numerical experiments demonstrating the influence of nonlinearities and the effect of the relaxation time. By analysing the process of formation of sharp and asymmetric profiles of temperature and displacement distributions, we showed that such profiles can be smoothed out with larger values 

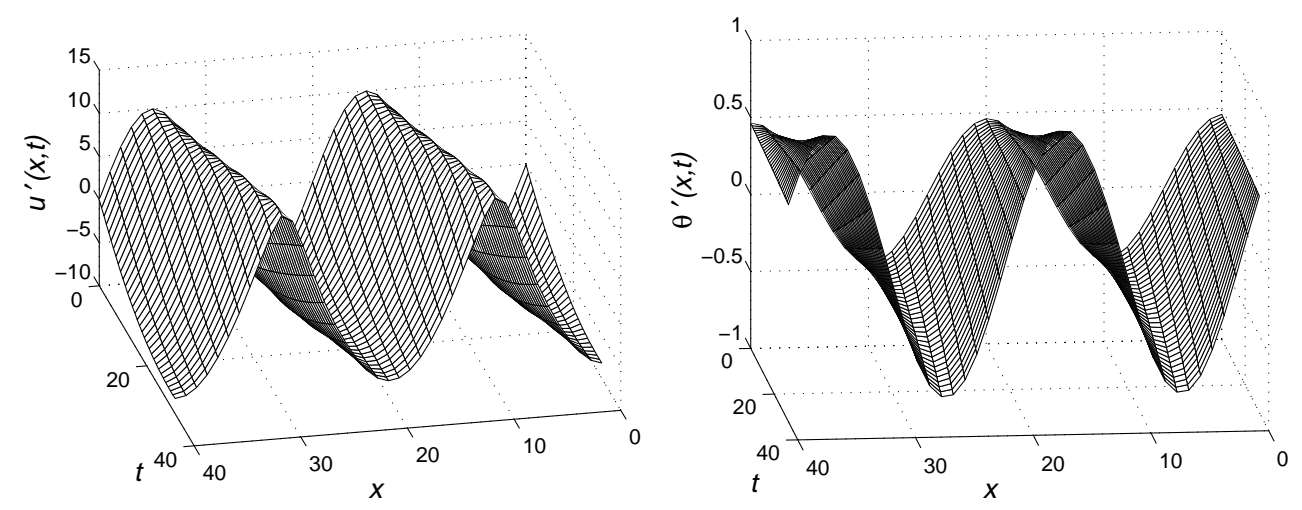

FiguRE 8: Displacement and temperature dynamics in the case of large relaxation times $(\tau=500)$.

of the relaxation time.

Acknowledgement: This research was supported by an Australian Research Council Grant.

\section{References}


[1] A. N. Abd-alla, A. F. Ghaleb, and G. A. Maugin. Harmonic wave generation in nonlinear thermoelasticity. Int. J. Engng. Sci, 32:1103-1116, 1994. C1359, C1360, C1362, C1365, C1370, C1372

[2] D. S. Chandrasekharaiah. Thermoelasticity with second sound: A review. Appl. Mech. Rev., 39:355-376, 1986. C1358, C1358, C1372

[3] D. S. Chandrasekharaiah. Hyperbolic thermoelasticity: A review of recent literature. Appl. Mech. Rev., 51:705-729, 1998. C1358, C1358

[4] J. Gawinecki. Global solution to the Cauchy Problem in non-linear hyperbolic thermoelasticity. Mathematical Methods in the Applied Sciences, 15:223-237, 1992.

[5] J. Gawinecki. Initial-boundary value problems in linear and nonlinear hyperbolic thermoelasticity theory. Z. Angew. Math. Mech., 78:S911-S912, 1998. C1360

[6] D. E. Glass and K. K. Tamma. Non-Fourier dynamic thermoelasticity with temperature-dependent thermal properties. J. of Thermophysics and Heat Transfer, 8:145-151, 1991. C1360

[7] A. E. Green and K. E. Lindsay. Thermoelasticity. J. Elasticity, 2:1-7 1972. C1359

[8] Ignaczak, J.. Soliton-like solutions in a nonlinear dynamic coupled thermoelasticity. J. Thermal Stresses, 13:73-98, 1990. C1358

[9] Jiang, S.. An uncoupled numerical scheme for the equations of nonlinear one-dimensional thermoelasticity. J. of Comput. and Applied Mathematics, 34:135-144, 1991. C1359 
[10] Kosinski, W.. Elastic waves in the presence of a new temperature scale. In Elastic Wave Propagation, pages 629-634. Elsevier, New York, 1989. C1358

[11] Kowalski, L.. Existence and uniqueness of the solution of the first boundary-initial value problem for linear hyperbolic thermoelasticity equations. Annales Societatis Mathematicae Polonae, Series I: Commentationes Mathematicae, 33:73-79, 1993. C1360

[12] Lasiecka, I.. Uniform dacay rates for full von Karman system of dynamic thermoelasticity with free boundary conditions and partial boundary dissipation. Commun. in PDE's, to appear. C1358, C1359, C1362, C1362

[13] Lax, P.D.. Development of singularities of solutions of nonlinear differential equations. J. Math. Physics, 5:611-613, 1964. C1359, C1372

[14] Lord, H.W. and Schulman, Y.. A generalized dynamic theory of thermoelasticity. J. Mech. Phys. Solids, 15:299-309, 1967. C1359

[15] MacCamy, R.C. and Mizel, V.J.. Existence and non-existence in the large of solutions of quasilinear wave equations. Archive for Rational Mechanics and Analysis, 25:299-320, 1967. C1359

[16] R. V. N. Melnik. Steklov's operator technique in coupled dynamic thermoelasticity. In R. W. Lewis and J. T. Cross, editors, Numerical Methods in Thermal Problems, Vol. X, pages 139-150. 1997. C1358

[17] J. H. Prevost and D. Tao. Finite element analysis of dynamic coupled thermoelasticity problems with relaxation times. J. Appl. Mech., 50:817-822, 1983. C1360 
[18] R. Racke. On the Cauchy Problem in nonlinear 3d-thermoelasticity. Math. Z., 203:649-682, 1990. C1358, C1359

[19] R. Racke. Blow-up in non-linear three-dimensional thermoelasticity. Mathematical Methods in the Applied Sciences, 12:267-273, 1990. C1358, C1359, C1372

[20] E. K. Rawy, L. Iskandar, and A. F. Ghaleb. Numerical solution of a nonlinear, one-dimensional problem of thermoelasticity. J. of Comput. and Applied Mathematics, 100:53-76, 1998. C1359, C1360, C1372

[21] M. Slemrod. Global existence, uniqueness, and asymptotic stability of classical smooth solutions in one-dimensional non-linear thermoelasticity. Archive for Rational Mechanics and Analysis, 76:97-133, 1981. C1359, C1360

[22] C. S. Suh and C. P. Burger. Effects of thermomechanical coupling and relaxation times on wave spectrum in dynamic theory of generalized thermoelasticity. J. of Appl. Mechanics, 65:605-613, 1998. C1360 